bind a plethora of factors that are essential for EPC function. However, these interactions are dependent upon specific structures of HS. We aim to establish whether structural changes of HS on EPCs underlie the age-associated functional deterioration of these cells. The number and function of EPCs in patients with systemic lupus erythematosus (SLE), a disease associated with accelerated vascular ageing, was compared with age-matched healthy controls (52 patients and 30 controls; mean age 52 and 50 years, respectively). To enumerate EPCs, mononuclear cells were labelled with CD133 and CD34 and analysed by flow cytometry. The formation of colony-forming units (CFUs) after 7 days in culture was a measure of EPC function. Cell surface HS structure was analysed using high performance liquid chromatography. While EPC levels did not significantly differ, impairment in EPC function with vascular ageing was evident from the significantly reduced mean number of CFU (7 $(\mathrm{SD}=5)$ vs 17 $(\mathrm{SD}=18), \mathrm{p}=0.01)$, with fewer large CFU $(17 \%$ vs $40 \%$; $\mathrm{p}<0.05)$ in patients than in controls. Preliminary data suggest decreased 2-Osulphation of $\mathrm{HS}$ in association with vascular age. Ongoing studies are investigating if this affects EPC migration, proliferation and integration into vascular structures. Proving our hypothesis will improve our understanding of age-associated endothelial dysfunction and ascertain whether EPCs and/or HS oligosaccharides have therapeutic potential to attenuate ageassociated vascular pathologies.

\section{BAS/ RELATIONSHIPS BETWEEN SLEEP DURATION AND VON BSCR53 WILLEBRAND FACTOR, FIBRINOGEN AND FACTOR VII: WHITEHALL II STUDY}

doi:10.1136/hrt.2010.205781.64

${ }^{1} \mathrm{M}$ A Miller, ${ }^{1} \mathrm{~N}$-B Kandala, ${ }^{2} \mathrm{M}$ Kumari, ${ }^{2} \mathrm{M} \mathrm{G}$ Marmot, ${ }^{1} \mathrm{~F}$ P Cappuccio. ${ }^{1}$ University of Warwick, Clinical Sciences Research Institute, UHCW Campus, WMS, Coventry, UK; ${ }^{2}$ Department of Epidemiology and Public Health, University College London, London, UK

Rationale Sleep is an emerging risk factor for cardiovascular disease (CVD). We examined the relationship between sleep duration and haemostatic factors important in CVD development, in a wellcharacterised occupational cohort.

Methodology The relationship between self-reported sleep duration and von Willebrand factor (vWF), fibrinogen and factor VII in 6400 individuals from the Whitehall II study was examined.

Results Owing to significant gender interactions $(p<0.001)$, the analysis was stratified by gender. After multiple adjustments, vWF levels were significantly higher in men with both short $(<6 \mathrm{~h}$ per night; 1.05 (95\% CI 1.01 to 1.08$)$ ) and long (>8 h per night; $1.05(95 \%$ CI 1.02 to 1.08)) duration of sleep compared with those who slept $7 \mathrm{~h}$ $(p<0.05$ for both). In women, levels of vWF were significantly higher in individuals who slept $>8 \mathrm{~h}(1.11$ (95\% CI 1.06 to 1.16$))$ than in those who slept for $7 \mathrm{~h}(\mathrm{p}<0.05)$. This difference was observed in premenopausal and postmenopausal women ( $p<0.05$ for each). The association seen in women appears to be non-linear $(p=0.02)$, but not in men $(p=0.09)$. No statistically significant associations between sleep duration and fibrinogen or factor VII were seen.

Conclusions Men sleeping for both short and long periods had higher vWF levels than those who slept for $7 \mathrm{~h}$. In women, there was a significant non-linear association with the highest levels mainly seen in long sleepers, irrespective of menopausal status. No major associations between sleep and either factor VII or fibrinogen were observed. Further longitudinal studies are required to fully investigate possible temporal relationships between sleep and vWF and the possible associated risk of CVD.

\section{BAS/ THE HYPOLIPIDAEMIC ACTIVITY OF NOVEL INDOLE-2- BSCR54 CARBOXAMIDES IN TRITON WR-1339-INDUCED HYPERLIPIDAEMIC RATS: A COMPARISON WITH BEZAFIBRATE}

doi:10.1136/hrt.2010.205781.65

G Shattat, T Al-Qirim, G Abu Sheikha, W El-Huneidi. Department of Clinical Sciences, Faculty of Pharmacy, Al-Zaytoonah Private University, Jordan

Using Triton WR-1339-induced hyperlipidaemic rats as an experimental model, we investigated whether compound 1 ( $\mathrm{N}$-(3-benzoylphenyl)$1 \mathrm{H}$-indole-2-carboxamide) and 2 (N-(4-benzoylphenyl)-1H-indole-2carboxamide) novel anti-hyperlipidaemic agents have any effect on plasma triglyceride, total cholesterol (TC) and high-density lipoprotein cholesterol levels (HDL-C) levels. Hyperlipidaemia was developed by intraperitoneal injection of Triton WR-1339 (200 mg/ $\mathrm{kg}$ body weight). At a dose of $15 \mathrm{mg} / \mathrm{kg}$ body weight, compounds 1 , 2 and bezafibrate (BF) significantly reduced the elevated plasma triglyceride levels after 7 and $24 \mathrm{~h}$. Furthermore, HDL-C levels were remarkably increased in all treated groups after 7 and $24 \mathrm{~h}$ compared with the hyperlipidaemic control group. However, only compounds 1 and 2-treated groups clearly showed a significant reduction in plasma TC levels after $24 \mathrm{~h}$. It is therefore reasonable to assume that compounds 1 and 2 may have a promising potential in the treatment of hyperlipidaemia and coronary heart diseases.

\section{BAS/ DIVERSE BACTERIA PROMOTE MACROPHAGE FOAM CELL BSCR55 FORMATION: POTENTIAL ROLE OF TOLL-LIKE RECEPTOR SIGNALLING PATHWAYS}

doi:10.1136/hrt.2010.205781.66

G Nicolaou, A H Goodall, C Erridge. Department of Cardiovascular Sciences, Glenfield Hospital, University of Leicester, Leicester, UK

We and others have shown that the molecular signatures of diverse bacterial species accumulate in human atheromatous lesions. Here, we aimed to determine the effect of non-viable bacteria on foam cell formation in vitro. Using human monocyte-derived macrophages and the murine $\mathrm{J} 774$ macrophage cell line, we found that any of a diverse panel of heat-killed Gram-positive or Gram-negative bacteria shown previously to accumulate in human atherosclerotic lesions promoted marked induction of foam cell formation in macrophages, as assessed by light-microscopy of Oil-red-O stained cells and Nilered-based flow cytometric quantification of cellular lipid accumulation. As Toll-like receptors (TLRs) have a central role in the induction of inflammatory signalling by bacteria, we next examined if specific TLR-ligands could also promote foam cell formation in the absence of intact bacteria. Remarkably, stimulation of macrophages with purified ligands specific for any of the TLRs (including lipopeptide, polyI:C, LPS, flagellin, ssRNA, loxoribine and CpG DNA) led to significant lipid accumulation. This process was not dependent on oxidation of low-density lipoprotein (LDL) as neither antioxidants nor the scavenger receptor blocker polyinosinic acid reduced foam cell formation. Moreover, the presence of LDL was not required for TLR-mediated foam cell formation. Specific inhibitors of TLR signalling prevented foam cell formation induced by TLR ligands or bacteria. We conclude that although the bacterial signatures present in human atheroma are likely to reflect non-viable, killed organisms, it remains possible that molecules derived from these organisms may promote the differentiation of macrophages to lipid-laden foam cells via mechanisms that are likely to include stimulation of TLRs. 